\title{
Gastric fistula secondary to drainage tube penetration: A report of a rare case
}

\author{
HUI-JIANG SHAO, BAO-CHUN LU, HUAN-JIAN XU, XIN-XIAN RUAN, JING-SONG YIN and ZHI-HONG SHEN \\ Department of Hepatobiliary Surgery, Shaoxing People's Hospital, Shaoxing Hospital of Zhejiang University, \\ Shaoxing, Zhejiang 312000, P.R. China
}

Received December 18, 2014; Accepted January 21, 2016

DOI: $10.3892 / \mathrm{ol} .2016 .4201$

\begin{abstract}
Cases of gastric fistula secondary to drainage tube penetration have rarely been reported. The current study presents a case of gastric penetration caused by misplacement of a drainage tube after a splenectomy. The patient was admitted to the Department of Hepatobiliary Surgery, (Shaoxing People's Hospital, Shaoxing Hospital of Zhejiang University, Shaoxing, Zhejiang, China) for blunt abdominal trauma due to injuries sustained in an automobile accident. A ruptured spleen was found and successfully removed surgically. On post-operative day 7, the patient complained of slight discomfort and tenderness in the left upper quadrant of the abdomen. In addition, $500 \mathrm{ml}$ of bile-colored fluid with small food particles was noted in the drainage tube. Barium $\mathrm{X}$-ray revealed a gastric fistula in the upper gastrointestinal tract. Gastroscopy indicated infiltration of the drainage tube into the gastric cavity. No significant peritoneal effusion was observed, as revealed by abdominal ultrasound examination. These results confirmed the diagnosis of a gastric fistula secondary to perforation by the drainage tube. Following conservative treatment with antibiotics and total parenteral nutrition, the general condition of the patient improved significantly. The drainage tube was withdrawn progressively, as the amount of fluid being discharged was decreasing. Gastroenterography confirmed perforation closure and the tube was finally removed on post-operative day 44.
\end{abstract}

\section{Introduction}

Abdominal drainage is widely used in abdominal surgery for the rapid evacuation of post-operative fluid collections. Gastric fistula, a common complication in gastrointestinal surgery, usually occurs due to the failure of anastomotic

Correspondence to: Dr Zhi-Hong Shen, Department of Hepatobiliary Surgery, Shaoxing People's Hospital, Shaoxing Hospital of Zhejiang University, 568 Zhongxing North Road, Shaoxing, Zhejiang 312000, P.R. China

E-mail: shensx0531@126.com

Key words: gastric fistula, drainage tube, penetration, perforation healing in gastrointestinal anastomosis and reconstruction, or in the greater curvature of the stomach with a direct injury to the gastric wall followed by splenectomy. The incidence of gastric fistula and patient outcome are dependent on surgery type (1) and clinicopathological factors (2). According to a recent meta-analysis, the incidence and mortality rates following laparoscopic sleeve gastrectomy are 2.2 and $0.11 \%$, respectively (3), whereas the incidence is $\sim 9.5 \%$ following gastrojejunostomy for the treatment of gastric carcinoma (4). There are various complications of gastric fistula, including abdominal pain, painful bowel obstruction and fever. In clinical practice, the diagnosis of gastric fistula is largely dependent on gastroenterography. The majority of gastric fistula cases may be treated by conservative therapy, including the administration of drugs such as somatostatin, antibiotics and supportive care (5). Certain cases may be treated using the injection of fibrin sealant (6), however, surgery should be performed in cases that have persisted for 120 days following diagnosis (7). Generally, patient outcome is good in patients with gastric fistula due to the administration of timely therapy (8). Gastric fistula secondary to the perforation by a drainage tube has rarely been reported $(9,10)$. In the present study, a case of gastric fistula caused by misplacement of a drainage tube following a splenectomy for abdominal trauma is reported.

\section{Case report}

A 54-year-old man was admitted to Shaoxing People's Hospital (Shaoxing Hospital of Zhejiang University, Shaoxing, Zhejiang, China) in April 2011 as an emergency due to injuries sustained in an automobile accident. A physical examination revealed tenderness over the left upper quadrant of the abdomen, with a blood pressure of $80 / 50 \mathrm{mmHg}$ (normal range, 90-140/60-90 $\mathrm{mmHg}$ ). Abdominal ultrasonography indicated splenic rupture and effusion in the peritoneal cavity. In the abdominocentesis, $\sim 4 \mathrm{ml}$ bloody liquid was extracted. Thus, a closed abdominal injury and abdominal hemorrhage was diagnosed. A laparotomy was performed in the Department of Hepatobiliary Surgery, which revealed a hemoperitoneum $(2,000 \mathrm{ml})$ and splenic rupture. A splenectomy was accomplished with no unexpected events and a rubber drainage tube was placed in the splenic recess in proximity to the gastric greater curvature. 
The patient was placed on a semi-liquid diet on post-operative day 4 . The post-operative drainage volume gradually decreased from $85 \mathrm{ml}$ on post-operative day 1 to $35 \mathrm{ml}$ on post-operative day 6 . On post-operative day 7 , the patient complained of slight discomfort and tenderness in the left upper quadrant of the abdomen. In addition, $\sim 50 \mathrm{ml}$ of purulent fluid was induced from the drainage tube. On post-operative day $9,500 \mathrm{ml}$ of bile-colored fluid with small food particles was noted in the drainage tube. Barium X-ray (BLF-15B X-ray Beam Limiting Device; Toshiba, Tokyo, Japan) revealed a gastric fistula in the upper gastrointestinal tract (Fig. 1). Gastroscopy (Evis Lucera CV-260SL; Olympus Corporation, Tokyo, Japan) indicated infiltration of the drainage tube into the gastric cavity (Fig. 2). No significant peritoneal effusion was observed, as revealed by abdominal ultrasound examination. The patient was consequently diagnosed with a gastric fistula secondary to perforation by the drainage tube. As a result, the drainage tube was withdrawn by $1 \mathrm{~cm}$.

The patient was treated with somatostatin $(6 \mathrm{mg} /$ day intravenously for a total of 21 days), total parenteral nutrition and broad-spectrum antibiotics according to the general principles of gastrointestinal fistula management (5). On post-operative day 36 , the drainage volume was decreased to $25 \mathrm{ml}$. Thus, the drainage tube was subsequently withdrawn by $2 \mathrm{~cm}$. No fluid was observed from the drainage tube on post-operative day 39. Upper gastroenterography (BLF-15B X-ray Beam Limiting Device) showed that the gastric perforation was closed and the drainage tube was removed on post-operative day 44 (Fig. 3). The patient was discharged, and no complications were noted during the 2 years of follow-up. Written informed consent was obtained from the patient for the publication of the present study.

\section{Discussion}

Drains are widely used in abdominal surgery in order to remove any collections or secretions and to act as a warning of hemorrhage or anastomotic leakage. The clinical complications of drainage tubes are infrequent, but may include abdominal infection, incisional herniation and prolapse of the abdominal contents (11). Among these complications, abdominal infection is most commonly noted. In addition, the threat of erosion into certain adjacent tissues is the rarest complication that may occur, with a risk of fistula development, particularly when drains are placed near upper digestive anastomoses and sutures (12). There have been a few reports of visceral injury with intestinal perforation or peritonitis due to suction drains that drew the bowel wall into the peripheral holes, or due to pressure necrosis by the tip of the drain $(13,14)$.

Several studies on gastric fistulae following splenectomy have been presented in the literature $(15,16)$. The condition was classically believed to be secondary to direct trauma to the gastric wall from surgical instrumentation. In addition, other induced factors, acting solely or in combination, may also predispose patients to the development of post-splenectomy gastric fistulae, including hematoma with an inflammatory reaction in the gastrosplenic omentum, generalized arteriosclerotic disease and the interruption of reflection of gastric

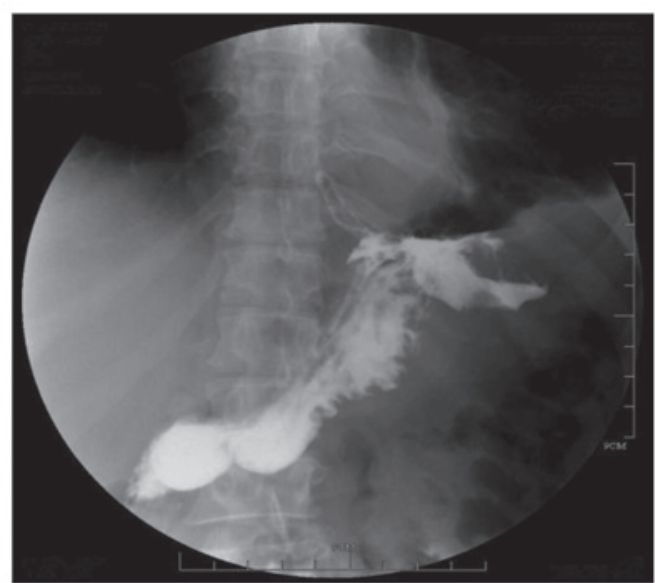

Figure 1. Gastric fistula noted upon upper gastroenterography.

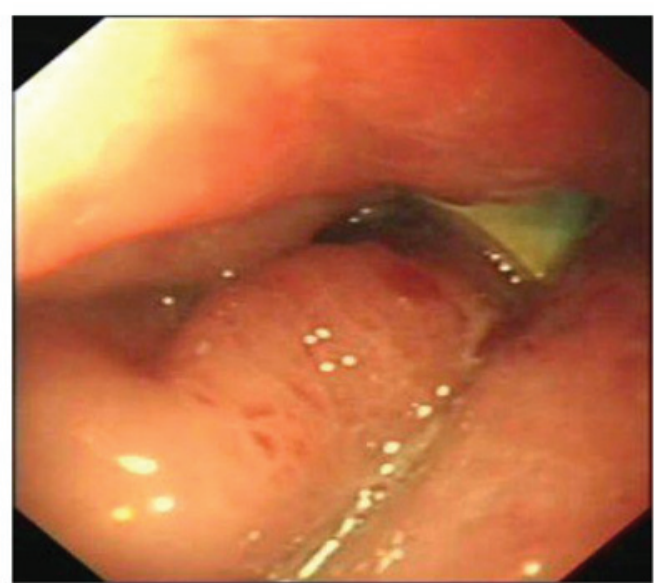

Figure 2. Drainage tube erosion of the gastric cavity, as identified using gastroscopy.

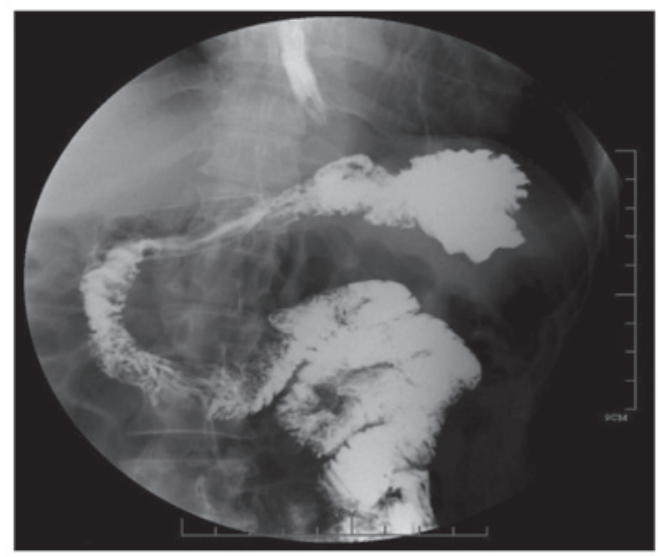

Figure 3. Closure of fistula following conservative management, as observed by gastroenterography.

muscle fibers into the gastrosplenic ligament (17). Notably, gastric penetration by drainage tube is extremely rare. In 2000 , only one case presented with a gastrocutaneous fistula following splenectomy, as a result of migration of the tube subsequent to placement (9). To the best of our knowledge, 
this is the second case of a patient who sustained drainage tube penetration of the stomach subsequent to a splenectomy.

The complication of a gastric fistula occurring due to penetration by a drainage system is associated with a number of factors, including the non-flexibility and stiffness of the drain devices, edema at the site of surgical manipulation and pressure necrosis of the stomach wall (18). In the present case, the drainage tube showed good biocompatibility, with a soft texture and superior elasticity. In addition, the surgery was performed by experienced staff. The onset of a gastric fistula caused by the erosion of the indwelling tube suggested that the misplacement and/or long-term placement of drains positioned close to the greater curvature of the stomach may be the main causative factor. Ischemic necrosis of the stomach wall occurred as a result of devascularization of the proximal stomach artery due to the long period of in situ compression by the tip of the drain. The gastric fistula was therefore induced with further infiltration of the tube into the gastric cavity.

Although it is always necessary to monitor the application of the drainage system, this precaution is of paramount importance in certain situations. In colonic anastomosis, only a 5\% sensitivity for the detection of anastomotic leaks has been recorded. On this basis, peritoneal drainage is not usually adopted by surgeons (19). When drainage is employed, it should be efficiently used and removed at the earliest safe time following the surgery. Durai et al suggested that it was relatively safe to remove a drainage tube if the drainage flow was $<25 \mathrm{ml}$ within $24 \mathrm{~h}$ (20). In addition, drains should be placed carefully to avoid direct contact with the major blood vessels, organs and anastomoses, and should be withdrawn and turned appropriately for a period of time (20).

Usually, the complication of drainage penetration with a fistula is treated using similar strategies to those for a gastric fistula with absent peritonitis. In the few available previous studies, patients have generally been treated conservatively by withdrawing the drains from the perforation site and permitting spontaneous healing of the fistula $(9,18)$. In the present case, the patient received somatostatin, total parenteral nutrition and broad-spectrum antibiotics, and recovered uneventfully. On the evidence currently available, conservative management may be feasible for patients without any signs of peritonitis, and the appropriate withdrawal of the drain from the perforation site is a prerequisite to ensure the success of this treatment strategy.

In conclusion, few cases of a gastric fistula secondary to perforation by a drainage tube have been reported. The present study describes one case of a gastric fistula that was caused by improper use of a drainage tube following a splenectomy for abdominal trauma. Following conservative treatment, the patient was discharged with a satisfactory outcome.

\section{References}

1. Smith MD, Adeniji A, Wahed AS, Patterson E, Chapman W, Courcoulas AP, Dakin G, Flum D, McCloskey C, Mitchell JE, et al: Technical factors associated with anastomotic leak after Roux-en-Y gastric bypass. Surg Obes Relat Dis 11: 313-320, 2015.
2. Kim SH, Son SY, Park YS, Ahn SH, Park do J and Kim HH: Risk factors for anastomotic leakage: A retrospective cohort study in a single gastric surgical unit. J Gastric Cancer 15: 167-175, 2015.

3. Parikh M, Issa R, McCrillis A, Saunders JK, Ude-Welcome A and Gagner M: Surgical strategies that may decrease leak after laparoscopic sleeve gastrectomy: A systematic review and meta-analysis of 9991 cases. Ann Surg 257: 231-237, 2013.

4. Liu BW, Liu Y, Liu JR and Feng ZX: Comparison of hand-sewn and stapled anastomoses in surgeries of gastrointestinal tumors based on clinical practice of China. World J Surg Oncol 12: 292, 2014.

5. Foster CE III and Lefor AT: General management of gastrointestinal fistulas. Recognition, stabilization and correction of fluid and electrolyte imbalances. Surg Clin North Am 76: 1019-1033, 1996

6. Kowalski C, Kastuar S, Mehta V and Brolin RE: Endoscopic injection of fibrin sealant in repair of gastrojejunostomy leak after laparoscopic Roux-en-Y gastric bypass. Surg Obes Relat Dis 3: 438-442, 2007.

7. Rebibo L, Bartoli E, Dhahri A, Cosse C, Robert B, Brazier F, Pequignot A, Hakim S, Yzet T, Delcenserie R, et al: Persistent gastric fistula after sleeve gastrectomy: An analysis of the time between discovery and reoperation. Surg Obes Relat Dis 12: 84-93, 2016.

8. Jacobsen HJ, Nergard BJ, Leifsson BG, Frederiksen SG, Agajahni E, Ekelund M, Hedenbro J and Gislason H: Management of suspected anastomotic leak after bariatric laparoscopic Roux-en-y gastric bypass. Br J Surg 101: 417-423, 2014.

9. Paraskevopoulos JA, Samoilis S, Papadakis G, Kostopoulos O and Kalimeris S: Drainage tube perforation of the stomach: An exceptionally rare complication. J Trauma 48: 330-331, 2000.

10. Rege S, Narlawar R, Deshpande A and Dalvi A: Iatrogenic gastric fistula due to inappropriate placement of intercostal drainage tube in a case of traumatic diaphragmatic hernia. J Postgrad Med 47: 108-110, 2001.

11. Kjossev KT and Losanoff JE: Complication of prophylactic intraperitoneal drainage. Dig Dis Sci 46: 2456, 2001.

12. González-Pinto I and González EM: Optimising the treatment of upper gastrointestinal fistulae. Gut 49 (Suppl 4): iv22-iv31, 2001

13. Nomura T, Shirai Y, Okamoto H and Hatakeyama K: Bowel perforation caused by silicone drains: A report of two cases. Surg Today 28: 940-942, 1998.

14. Nikolić M, Karthikesalingam A, Nachimuthu S, Tang TY and Harris AM: Biliary peritonitis caused by a leaking T-tube fistula disconnected at the point of contact with the anterior abdominal wall: A case report. J Med Case Rep 2: 302, 2008.

15. Zheng X, Yao Y and Liu Q: Gastric perforation after laparoscopic splenectomy and esophagogastric devascularization for portal hypertension: Report of a case. Surg Laparosc Endosc Percutan Tech 21: e209-e212, 2011.

16. Martinez CA, Waisberg J, Palma RT, Bromberg SH, Castro MA and Santos PA: Gastric necrosis and perforation as a complication of splenectomy. Case report and related references. Arq Gastroenterol 37: 227-230, 2000.

17. Harrison B, Glanges E and Sparkman RS: Gastric fistula following splenectomy: Its cause and prevention. Ann Surg 185: 210-213, 1977.

18. Eleftheriadis E: Drainage-tube penetration into the gastric lumen, mimicking a high-volume enterocutaneous fistula. The significance of postoperative endoscopy. Surg Endosc 4: 184-185, 1990.

19. Puleo FJ, Mishra N and Hall JF: Use of intra-abdominal drains. Clin Colon Rectal Surg 26: 174-177, 2013.

20. Durai R, Mownah A and Ng P: Use of drains in surgery: A review. J Perioper Pract 19: 180-186, 2009. 